\title{
A importância da fisioterapia no atendimento de pessoas com Transtorno do
}

\section{Espectro Autista}

\author{
The importance of physiotherapy in the care of people with Autism Spectrum Disorder \\ La importancia de la fisioterapia en la atención de personas con Trastorno del Espectro Autista
}

Recebido: 12/04/2021 | Revisado: 17/04/2021 | Aceito: 20/04/2021 | Publicado: 05/05/2021

Lucas Gabriel de Araújo Marcião

ORCID: https://orcid.org/0000-0002-2592-8644

Centro Universitário da Amazônia, Brasil

E-mail: lucasgabrielaraujomarciao90@gmail.com

Grace Ellen Pereira Costa

ORCID: https://orcid.org/0000-0001-5101-4152

Universidade Federal do Pará, Brasil

E-mail: grace123.stm@gmail.com

Patricia Emerich Lima

ORCID: https://orcid.org/0000-0003-3736-840X

Centro Universitário da Amazônia, Brasil

E-mail: patrícia.27emerick@hotmail.com

Victor Hugo Filgueiras da Silva

ORCID: https://orcid.org/0000-0002-2554-3496

Centro Universitário Doutor Leão Sampaio, Brasil

E-mail: victorhugofs@outlook.com.br

Ana Beatriz Bezerra

ORCID: https://orcid.org/0000-0002-0099-8427

Centro Universitário Doutor Leão Sampaio, Brasil E-mail: beatrizbezerra.542@gmail.com

Ana Carla Calixto Oliveira

ORCID: https://orcid.org/0000-0001-6611-0905

Centro Universitário Doutor Leão Sampaio, Brasil

E-mail: ana.carla.ac@hotmail.com

Leandro Ferreira de Moura

ORCID: https://orcid.org/0000-0001-8772-2089

Centro Universitário Pitágoras de Fortaleza, Brasil

E-mail: leandromour2@gmail.com

Ana Rita Pereira Cobalchini

ORCID: https://orcid.org/0000-0001-5628-6982

Universidade do Estado do Pará, Brasil

E-mail: cobalchinir@gmail.com

Manoel Leonardo Tavares da Silva

ORCID: https://orcid.org/0000-0003-2261-0283

Centro Universitário UniFacid, Brasil

E-mail: leo.tavares3333@gmail.com

Danielle Gomes de Sousa

ORCID: https://orcid.org/0000-0001-6576-5918

Universidade Federal do Piauí, Brasil

E-mail: dannyfirmino98@gmail.com

Izabel Anna da Silva Rocha

ORCID: https://orcid.org/0000-0001-8241-4108

Centro Universitário Doutor Leão Sampaio, Brasil

E-mail: izabelanna.2@hotmail.com

Ricardo Nobre dos Santos

ORCID: https://orcid.org/0000-0003-4606-1223

Universidade Cruzeiro do Sul, Brasil

E-mail: ricardonobresantos@hotmail.com

\begin{abstract}
Resumo
O Transtorno do Espectro Autista (TEA) é um distúrbio do neurodesenvolvimento que pode comprometer a comunicação de maneira verbal ou não verbal, com manifestação de movimentos repetitivos e momentos de distanciamento do contato com a realidade. Assim, é fundamental a inclusão do tratamento fisioterapêutico precocemente para desenvolver as funções das atividades de rotina, como também a melhoria da evolução do desenvolvimento da coordenação e interação interpessoal. O objetivo desse trabalho é discutir a importância da atuação do profissional fisioterapeuta no atendimento de pessoas com Transtorno do Espectro Autista. Trata-se de uma revisão
\end{abstract}


narrativa de literatura. Para este artigo foram feitas buscas manuais na plataforma Google Scholar e selecionados artigos pertinentes à temática para composição das referências. A partir dos dados apresentados, identifica-se que o papel do fisioterapeuta no atendimento de pessoas com TEA é possibilitar melhorias tanto motoras como mentais a partir de técnicas que estimulem a proximidade ao paciente, diálogo, integração social e trabalho de independência do paciente de maneira lúdica. Assim, primeiro no que se refere ao estabelecimento motor, existe a construção comunicativa que define a relação de confiança com o paciente. Por fim, compreende-se a importância da atuação do fisioterapeuta no atendimento ao paciente com TEA, dado a necessidade de estímulos motores e sensoriais. É importante ressaltar a necessidade de intervenção precoce para melhores respostas ao tratamento e adaptação ao espaço. Dessa maneira, a evolução nos aspectos motores e sensoriais auxiliam na melhoria da qualidade de vida e integração social dos indivíduos. Palavras-chave: Fisioterapia; Transtorno do Espectro Autista; Promoção da saúde.

\begin{abstract}
Autism Spectrum Disorder (ASD) is a neurodevelopmental disorder that can compromise communication verbally or nonverbally with manifestation of repetitive movements and moments of distancing from contact with reality. Thus, it is essential to include physical therapy treatment early to develop the functions of routine activities, as well as improving the evolution of the development of coordination and interpersonal interaction. The aim of this work is to discuss the importance of the physical therapist professional's role in the care of people with Autism Spectrum Disorder. This is a narrative literature review. For this article were made manual searches on the Platform Google Scholar and selected articles relevant to the theme for the composition of references. From the data presented it is identified that the role of the physiotherapist in the care of people with ASD is to enable both motor and mental improvements based on techniques that stimulate proximity to the patient dialogue, social integration and work of independence of the patient in a playful way. Thus, first as regards the motor establishment there is a communicative construction that defines the relationship of trust with the patient Finally, it is understood the importance of the physiotherapist's role in the care of patients with ASD, given the need for motor and sensory stimuli. It is important to highlight the need for early intervention for better responses to treatment and adaptation to space. In this way, the evolution in the motor and sensorial aspects helps to improve the quality of life and social integration of the individuals.
\end{abstract}

Palavras-chave: Physical therapy specialty; Autism Spectrum Disorder; Health promotion.

\title{
Resumen
}

El trastorno del espectro autista (TEA) es un trastorno del neurodesarrollo que puede comprometer la comunicación verbal o no verbal, con movimientos repetitivos y momentos de distancia del contacto con la realidad. Por ello, es fundamental incluir la fisioterapia temprana para desarrollar las funciones de las actividades rutinarias, así como para mejorar la evolución del desarrollo de la coordinación y la interacción interpersonal. El objetivo de este trabajo es discutir la importancia del papel del fisioterapeuta profesional en el cuidado de personas con Trastorno del Espectro Autista. Esta es una revisión de literatura narrativa. Para este artículo se realizaron búsquedas manuales en la plataforma Google Scholar y se seleccionaron artículos relevantes al tema para la composición de las referencias. A partir de los datos presentados, se identifica que el papel del fisioterapeuta en la atención de las personas con TEA es posibilitar mejoras tanto motoras como mentales basadas en técnicas que fomenten la proximidad al paciente, el diálogo, la integración social y la independencia del paciente trabajan de forma forma diferente juguetona. Así, primero en lo que se refiere al establecimiento motor, está la construcción comunicativa que define la relación de confianza con el paciente. Finalmente, se entiende la importancia del desempeño del fisioterapeuta en el cuidado de pacientes con TEA, dada la necesidad de estímulos motores y sensoriales. Es importante enfatizar la necesidad de una intervención temprana para mejores respuestas al tratamiento y adaptación al espacio. De esta forma, la evolución en los aspectos motor y sensorial ayuda a mejorar la calidad de vida y la integración social de los individuos.

Palavras chave: Fisioterapia; Trastorno del Espectro Autista; Promoción de la salud.

\section{Introdução}

O Transtorno do Espectro Autista (TEA) é definido como um distúrbio do neurodesenvolvimento que compromete aspectos como os contatos sociais, a interação social e o comportamento (Fernandes et al, 2020). Além disso, é um transtorno invasivo que pode afetar a comunicação na forma verbal ou não verbal, com manifestação de movimentos repetitivos e momentos de distanciamento do contato com a realidade (Dos Santos et al, 2018).

O autismo pode ter inúmeras origens, sendo a mais discutida entre os especialistas da neurologia, a qual os sinais e sintomas são decorrentes de um desvio no transporte de informações entre os lobos cerebrais. Dessa forma, o indivíduo com TEA deve fazer terapia para que exista a promoção de novas experiências do sentido com propósito de suprir a necessidade de estímulos sensoriais (BOSE, 2016). O diagnóstico do TEA é realizado de maneira exclusivamente clínica e com embasamento no Manual Estatístico e Diagnóstico da Associação Americana de Psiquiatria, em que é feita análise em níveis que alegam a 
comprovação do autismo (Brunoni, 2014). Há necessidade de observação dos sinais do espectro até às evidências clínicas concretas, para isso é preciso acompanhamento que segue as diretrizes do atraso no desenvolvimento (Mousinho, 2016).

Sendo assim, o autismo não é uma doença, mas um transtorno do espectro autista, em que é estabelecido uma linha de pensamento comportamental com causas e graus de complexidade, umas das características marcantes dos portadores de TEA são déficits na interação social e na comunicação, com sequências de movimentos repetitivos e estereotipados (Klin, 2006). Dessa maneira, existem três graus do Espectro: Primeiro, grau leve com presença de dificuldade para interação social, mas possui relativa adaptação ao meio externo, porém, apresenta características do TEA e suas dificuldades; segundo, considerados pacientes com dificuldade de comunicação necessitando de um tratamento mais especializado; terceiro, são identificados por não conseguir se adaptar a ambientes externos, apresentam muitos movimentos repetitivos e precisam de atenção intensiva no seu tratamento (Silva \& Mulick, 2009).

Com diagnóstico comprovado de TEA é de suma importância iniciar um acompanhamento e intervenção multiprofissional com capacidade para desenvolver uma melhora na qualidade de vida desses pacientes. Dessa maneira, é fundamental a inclusão do tratamento fisioterapêutico precocemente no autismo para desenvolver as funções das atividades de rotina, como também a melhoria da evolução do desenvolvimento da coordenação e interação interpessoal, à vista disso conduz uma boa qualidade de vida de portadores de Autismo (Azevedo \& Gusmão, 2016).

Dessa maneira, identifica-se como objetivo desse trabalho, discutir a importância da atuação do profissional fisioterapeuta no atendimento de pessoas com Transtorno do Espectro Autista.

\section{Método}

Trata-se de uma revisão narrativa de literatura, cuja função é permitir que o leitor possa se atualizar e obter informações acerca de um determinado assunto em curto período de tempo. Assim, consiste em uma publicação que contempla o "estado da arte" de temáticas (Rother, 2007). Para este artigo foram feitas buscas manuais na plataforma Google Scholar e selecionados artigos pertinentes à temática para composição das referências.

\section{Resultados e Discussão}

Pessoas com TEA apresentam retardo nas conexões que são responsáveis por dar sequência a movimentos e ações rotineiras, podem manifestar dificuldade de realizar ou aprender habilidades de coordenação como desenho e escrita. Nesse contexto, pode existir um impacto negativo na realização de afazeres diários ou atividades como a prática de determinado esporte (Geschwind, 2013; Rosa Neto et al, 2013). O TEA pode apresentar diferentes manifestações no indivíduo, como: tensão muscular, hipotonia, posições e atitudes, problemas na marcha, atraso nos efeitos motores dos processos mentais e estereotipais. Dessa forma, o fisioterapeuta deve intervir com técnicas para que o portador tenha aquisição de experiências sensório motor, a fim de contribuir para a evolução adaptativa e interação social, desencadeando uma interrelação corpo e mente (Azevedo \& Gusmão, 2016).

A partir do diagnóstico de TEA ser definido é importante implementar o tratamento multidisciplinar ao indivíduo, dado que são aspectos variados que atingem o paciente e portanto, a atuação de uma equipe multiprofissional pode melhorar o desempenho e qualidade de vida dos portadores. Essa equipe é composta por profissionais como: terapeutas ocupacionais, psicólogos, fonoaudiólogos (Dos Santos, et al). Nessa perspectiva, o papel do fisioterapeuta será possibilitar melhorias tanto motoras como mentais a partir de técnicas que estimulem a proximidade ao paciente, diálogo, integração social e trabalho da independência do paciente de maneira lúdica (Fernandes et al, 2020).

O profissional fisioterapeuta consegue com formas terapêuticas físicas, atuar na prevenção e melhoria de situações patológicas. Esse trabalho acontece por avaliações musculoesqueléticas, relacionadas à ergonomia, diagnósticos, aplicação de 
exames, prescrição e planejamento. Atuando, assim, na promoção da qualidade de vida e reabilitação do paciente (Maia et al, 2015). Dado isso, no cenário do atendimento a pacientes com TEA, o fisioterapeuta tem papel essencial no tratamento dos acometimentos motores e na prevenção de agravos ao estado do paciente (De Mendonça, 2020).

A atuação do fisioterapeuta ao melhorar a função motora, compreende também a melhoria do aspecto interação social relacionado a esses pacientes, quando associada a estimulações em saúde e educação. Assim, primeiro ao estabelecimento motor, existe a construção comunicativa que define a relação de confiança com o paciente. Podem ser citados como exemplos dessa interação: o contato visual, o conforto com o toque com intuito de aporte físico, comunicação verbal e a partir de gestos (De Mendonça, 2020).

Assim, o fisioterapeuta atua para melhoria das seguintes características que podem atingir pessoas com TEA:

\section{Estereotipias}

É definido como a particularidade de expressar sentimentos por meio de movimentos, com a finalidade de uma busca de uma sensação de expressão de si próprio (Geschwind, 2013; Pfeiffer et al., 2013). Nesse contexto, as emoções ligadas aos movimentos estereotipadas realizados por pessoas portadoras de TEA na infância devem ser conhecidas como movimentos autísticos, visto que não são direcionados a alguém, as movimentações são umas das principais portas de entradas para o início do tratamento (Levin, 2000).

\section{Tônus muscular}

Em portadores de TEA na fase infantil, inúmeras vezes, é complicado a avaliação do tônus muscular de forma única. A hipotonia muscular pode acarretar modificações da coluna vertebral como uma escoliose na adolescência (Geschwind, 2013, Sacrey et al, 2014). Nesse pensamento, a estabilidade ou a instabilidade de tensão muscular, possuem suas particularidades ou seus obstáculos que irão atingir a vivência do paciente (Fernandes, 2002).

A aptidão motora, intelectual e afetiva agrega estabelecer relações com a sociedade, a partir disso é fortalecido as estimulações que o meio e as pessoas lhe impõem (Ferreira, 2000). O trabalho da fisioterapia é fundamental no atendimento precoce do autismo, com foco nas técnicas para a plasticidade cerebral, e ajudando de forma eficaz na construção e melhora da qualidade de vida, sendo liberado ao indivíduo com TEA uma inclusão social (Silva, 2009).

\section{Marcha}

Quando acontece o diagnóstico tardio em indivíduos com TEA, o paciente pode apresentar irregularidades no padrão de ciclo de marcha, como a necessidade de ficar na ponta dos pés, diferença na proporção do braço durante o movimento de caminhada e anomalias em todo o movimento, é considerado que a irregularidade dos membros superiores pode ter relação com a tentativa de se manter equilibrado (Fernandes, 2013). O fisioterapeuta deve estar apto com conhecimentos sobre o desenvolvimento das habilidades motoras normais e anormais e tentar entender o autista e o seu mundo, criando uma relação com vivências sensório motor.

\section{Desenvolvimento neuropsicomotor}

$\mathrm{O}$ atraso no desenvolvimento sensório motor exige etapas no tratamento como o modo de aprender e interpretar a si mesmo, aprendizagem social e da linguagem, diminuir a rigidez e as estereotipias, dissolver comportamentos mal adaptativos e aumentar o bem-estar da família. Sendo assim, é indubitável para uma boa relação com os portadores utilizar de métodos criativos, seja por jogo de sinais ou dispositivos eletrônicos projetados para o tratamento de autismo (Alessandri, 2005). 
O papel da fisioterapia é por meio do tratamento melhorar o convívio social, trabalhando técnicas de concentração com uso de aparelhos pedagógicos, e diminuir os movimentos anormais. Assim, pode contribuir para a melhora e o autoconhecimento corporal, além do treino de habilidades motoras, equilíbrio e coordenação de pessoas com TEA (Marques, 2002).

\section{Considerações Finais}

A partir desse artigo, compreende-se a importância da atuação do fisioterapeuta no atendimento ao paciente com TEA, dado a necessidade de estímulos motores e sensoriais. É importante ressaltar a necessidade de intervenção precoce para melhores respostas ao tratamento e adaptação ao espaço. As atividades que são trabalhadas devem ser lúdicas que associam a coordenação e o equilíbrio. A evolução nos aspectos motores e sensoriais auxiliam na melhoria da qualidade de vida e integração social dos indivíduos.

Em virtude disso é de extrema relevância a identificação precoce do transtorno do espectro autista e a necessidade do diagnostico diferencial, a capacitação e a atualização das técnicas dos profissionais fisioterapeutas que realizam tratamentos de pessoas com TEA, por a fisioterapia ser comprometida com a saúde e ser atuante na promoção, prevenção, recuperação e reabilitação desses pacientes, sendo assim, o governo deve garantir o acesso digno das pessoas autistas, pois o individuo vai precisar de auxilio no seu desenvolvimento, com isso, terá que ser elaborador meios para construção de diretos de cidadania e inserção social.

\section{Referências}

Alessandri, M. (2005). Podemos curar el autismo? Del desenlace clínico a la intervención. Revista de Neurologia, 40(1), $131-136$.

Azevedo, A. \& Gusmão, M. (2016). A importância da fisioterapia motora no acompanhamento de crianças autistas. Revista Eletrônica Atualiza Saúde, 2(2), 76-83.

Brasil. (2014). Diretrizes de Atenção à Reabilitação da Pessoa com Transtornos do Espectro do Autismo (TEA).

https://bvsms.saude.gov.br/bvs/publicacoes/diretrizes_atencao_reabilitacao_pessoa_autismo.pdf.

Bosa, C., Zanon, R. \& Backes, B. (2016). Autismo: construção de um Protocolo de Avaliação do Comportamento da Criança-Protea-R. Revista Psicologia: Teoria e Prática, 18(1), 194-205.

Brunoni, D. (2014). Diagnóstico etiológico dos transtornos do espectro do autismo: quando e quais exames pedir. Seção de Pôster apresentado no $2^{\circ}$ Congresso Internacional Sabará de Especialidades Pediátricas, São Paulo, SP.

De Mendonça, F. S. et al. (2015). Desenvolvimento da Criança e do Adolescente: Evidências Científicas e Considerações Teórica s-Práticas: As principais alterações sensório-motoras e a abordagem fisioterapêutica no transtorno do espectro autista. In: Editora Científica, Desenvolvimento da Criança e do Adolescente: Evidências Científicas e Considerações Teóricas-Práticas. São Paulo, SP: Editora Científica.

Dos Santos, L. F. et al. (2017). Estudo das Principais Contribuições da Fisioterapia em Pacientes com Transtorno do Espectro Autista (TEA) diagnosticados. Seção de Pôster apresentado no Congresso de Ensino, Pesquisa e Extensão da UEG, Goiás, GO.

Fernandes, C. R.; Souza, W. Á. A. A. D.; Camargo, A. P. R. (2020). Influência da Fisioterapia no Acompanhamento de Crianças Portadoras do TEA (Transtorno do Espectro Autista). Revista de Ciências da Saúde e Sociais Aplicadas do Oeste Baiano, 5(1), $52-68$.

Fernandes, F. S. (2008). O corpo no autismo. Revista da Vetor Editora, 9(1), 109-114.

Ferreira, C. A. M. (2000). Psicomotricidade, da educação infantil à gerontologia: teoria e prática. Lovise.

Geschwind, D. (2013). Avanços no Autismo. Revista de Medicina, 60(1), 367-380.

Klin, A. (2006). Autismo e Síndrome de Asperger: uma visão geral. Revista Brasileira de Psiquiatria, 28(1), 56-60.

Levin, E. (2000). A clínica psicomotora: o corpo na linguagem. Vozes.

Marques, T. (2002). Autismo: que intervenção? Revista cidade solidária, 8(3), 99-104.

Maia, F. E. S. et al.(2015) A importância da inclusão do profissional fisioterapeuta na atenção básica de Saúde. Revista da Faculdade de Ciências Médicas de Sorocaba,17(3), 110-115.

Mousinho, R. \& Navas, A. (2016). Mudanças apontadas no DSM-5 em relação aos transtornos específicos de aprendizagem em leitura e escrita. Revista Debates Em Psiquiatria, 46 (38) 
Research, Society and Development, v. 10, n. 5, e24410514952, 2021

(CC BY 4.0) | ISSN 2525-3409 | DOI: http://dx.doi.org/10.33448/rsd-v10i5.14952

Prates, A. C. et al. (2019) Os benefícios da fisioterapia na independência funcional em crianças com transtorno do espectro autista. Revista Eletrônica do Centro Universitário Católico Salesiano Auxilium, 4 (4), 79-86.

Pfeiffer, B. et al. (2013). Effectiveness of Sensory Integra- tion Interventions in Children With Autism Spectrum Disorders: A Pilot Study. Autism Speaks. Funded Manuscripts, Canadá, 65(1), 76-85.

Rother, E. T. (2017). Revisão sistemática X revisão narrativa. Acta Paulista de Enfermagem, 20(2), 5-6.

Neto. F. R. et al. (2013). Efeitos da intervenção motora em uma criança com transtorno do Espectro do Autismo. Temas sobre Desenvolvimento, 3(1), 110114.

Silva, M., \& Mulick, J. A. (2009). Diagnosticando o transtorno autista: aspectos fundamentais e considerações práticas. Psicologia: Ciência e Profissão, 29(1), 116-131.

Sociedade brasileira de pediatria. (2019). Manual de orientação: Transtorno do Espectro do Autismo. https://www.sbp.com.br/fileadmin/user_upload/21775cMO_-_Transtorno_do_Espectro_do_Autismo.pdf. 